\title{
Consideraciones sobre la capa de ozono y su relación con el cáncer de piel
}

Francisca Sánchez C.

\author{
The relationship between \\ the ozone layer and skin cancer
}

\begin{abstract}
In the recent decades, a sustained increase in the worldwide incidence of skin cancer has been observed and Chile is not the exception. The most important risk factor is the exaggerated and repeated exposure to ultraviolet radiation coming from the sun. The ozone layer restricts the transmission of type B and C ultraviolet light. Since 1980, a sustained depletion of stratospheric ozone levels is occurring, specially in middle latitudes (-30 to $\left.-60^{\circ}\right)$. Along with this depletion, the amount of ultraviolet light that reaches the earth surface is increasing. This article reviews some basic concepts about the ozone layer and the association between its depletion and skin cancer. The general population should be informed about the risks of inadequate and exaggerated exposure to sunlight (Rev Méd Chile 2006; 134: 1185-90).

(Key words: Ozone; Skin neoplasms; Sun light; Ultraviolet rays).
\end{abstract}

\section{Recibido el 13 de septiembre, 2005. Aceptado el 26 de enero, 2006. \\ Servicio de Dermatología, Clínica Alemana. \\ Facultad de Medicina, Clínica Alemana-Universidad del Desarrollo.}

L a incidencia de cáncer de piel ha aumentado notoriamente en todo el mundo, siendo en Estados Unidos de Norteamérica (USA) y Australia el cáncer más frecuente de todo el organismo ${ }^{1}$. En Europa occidental, la incidencia de cáncer de piel es aproximadamente igual a la de cáncer de pulmón o de mama ${ }^{2}$. En nuestro país hay pocos estudios epidemiológicos al respecto y en ellos también se observa la misma tendencia. Un estudio, en el que se analizó retrospectivamente la incidencia de cánceres de piel en cuatro hospitales del área metropolitana, durante el periodo comprendido entre 1992 y 1998, se encontró que las tasas de incidencia de cáncer cutáneo global aumentaron $43 \%$ en dicho periodo ${ }^{3}$. Otros estudios, también en la región metropolitana, Hospital

Correspondencia a: Dra. Francisca Sánchez Clarke. Unidad de Dermatología, Clínica Alemana, Santiago, Chile. Fono: 2101444. Fax: 2101036. E mail: fsanchez@alemana.cl
Sótero del Río y de la XII región, en Punta Arenas, mostraron una tendencia similar ${ }^{4,5}$.

Se acepta universalmente que la exposición a la radiación solar es el factor de riesgo más importante para el desarrollo de cáncer cutáneo, especialmente el cáncer de piel no melanoma y que la radiación ultravioleta presente en la radiación solar es el principal factor etiopatogénico ${ }^{6,7}$.

La cantidad y la composición de radiación ultravioleta solar que llega a la superficie de la tiema, depende de varios factores, como el ángulo del cenit solar que varía durante el día, la latitud, altitud, estación del año, hora del día, presencia de nubes, la polución y la concentración de ozono estratosférico ${ }^{8}$. De todos los factores antes mencionados, el ozono de la estratosfera es el factor más importante en determinar la cantidad de radiación carcinogénica que recibimos. Sin embargo, la disminución de la capa de ozono no es el factor más gravitante para explicar el aumento en la incidencia de cáncer cutáneo observado, sino la exposición exagerada al sol ${ }^{9-11}$. 
El propósito de este artículo es entonces, entregar algunas nociones acerca de la capa de ozono y su relación con cáncer de piel, para que todos los facultativos puedan contribuir informadamente con la educación de la población en esta materia.

\section{CONCEPTOS GENERAIES SOBRE EL OZONO Y CAPA DE OZONO}

La atmosfera está compuesta mayoritariamente por nitrógeno (78\%) y en segundo lugar por oxígeno (26\%). El porcentaje restante corresponde a numerosos gases traza, entre los cuales se encuentra el ozono, en cantidades ínfimas de pocas moléculas por millón de partículas de aire $(0,01 \%)$. Sin embargo, cumple un rol esencial en la conservación de la vida en el planeta tal como la conocemos, pues nos protege de la radiación ultravioleta que es el carcinógeno físico más importante para el hombre, animales y ecosistemas marinos ${ }^{12}$.

El ozono es una molécula formada por tres átomos de oxígeno y se crea en dos lugares de la atmosfera. Noventa por ciento o más del ozono se produce en la parte alta de la estratosfera, a $50 \mathrm{~km}$ de la superficie terrestre y comesponde al ozono benéfico, protector de la radiación ultravioleta. Diez por ciento del ozono se produce en las grandes ciudades, a nivel de la superficie terrestre o troposfera y es un componente del smog. Este ozono troposférico es muy imitativo de las vías aéreas, conjuntivas y pulmones $^{12}$. La acción benéfica del ozono de la estratosfera se debe, en primer lugar, a que tiene la propiedad de absorber selectivamente longitudes de onda en el rango de la radiación ultravioleta. La radiación ultravioleta se ha dividido arbitrariamente en: UVC (longitudes de onda menores a $280 \mathrm{~nm}$ ), UVB (280 nm - $315 \mathrm{~nm})$ y UVA $(315 \mathrm{~nm}-400 \mathrm{~nm})^{2}$. El ozono de la estratosfera absorbe 100\% de UVC, bloquea el paso de alrededor de $90 \%$ de UVB y no afecta para nada la transmisión de UVA ${ }^{13}$. En segundo lugar, el rol beneficioso del ozono se debe a que $90 \%$ o más del ozono estratosférico se concentra entre los 15 y $30 \mathrm{~km}$ de altitud, formando lo que llamamos capa de ozono. Como se mencionó, el ozono se produce a $50 \mathrm{~km}$ de la superficie de la tierra, pero las comientes de la estratosfera lo desplazan y concentran a menor altura, lo que permite optimizar la capacidad de filtrar las radiaciones a su paso por la atmosfera ${ }^{12}$.

El término "columna de ozono" se refiere a cuántas moléculas de ozono hay en el aire sobre cierto punto en la tierra y esta cantidad se expresa en "unidades Dobson" (UD).

Si se trajera la capa de ozono a la superficie de la tierra a temperatura y presión estándar, ésta medińa 3 $\mathrm{mm}$ de grosor. A esta medida de $3 \mathrm{~mm}$, se le asignó, arbitraniamente, el valor de 300 UD que corresponde, aproximadamente, al promedio global de la columna de ozono ${ }^{12,13}$. En los últimos treinta años, el ozono de la estratosfera ha sido estudiado con dedicación y una caractenística importante de la capa de ozono, es que tiene un comportamiento extremadamente dinámico y su grosor vanía constantemente. Por este motivo, el valor promedio de 300 UD debe ser considerado como un marco de referencia, ya que los niveles de la columna de ozono dependen ${ }^{14}$ de: a) la ubicación geográfica: la capa de ozono es más delgada en el Ecuador que en latitudes medias y, en general, en el hemisferio sur los valores de la columna de ozono son más bajos que en el hemisferio norte; b) las estaciones: los niveles más bajos se presentan a fines del inviemo e inicio de primavera y los más altos, a fines del verano e inicio de otoño en ambos hemisferios. Los registros más bajos de todo el planeta se dan sobre la Antártica en los meses de septiembre y octubre; c) fenómenos naturales: actividad solar, explosiones volcánicas que aportan gases que destruyen el ozono y las comientes de aire de la estratosfera, responsables del desplazamiento de las moléculas de ozono.

Por esta variabilidad, que es un atributo natural, las mediciones aisladas de los valores de la columna de ozono en un tiempo y ubicación particular, no son tan importantes. Lo fundamental es determinar las grandes tendencias, lo que implica registrar los valores de ozono durante largos períodos de tiempo.

También hemos aprendido en estos años, cómo el hombre ha alterado el equilibrio ecológico de la capa de ozono con la producción y emisión a la atmosfera de las llamadas "sustancias depletoras de ozono" (SDO). Las más conocidas son los clorofluorocarbonos (CFC), que se usaron en la fabricación de aerosoles, refrigeradores, equipos de aire acondicionado. Son muy reactivos, una molécula de cloro puede destruir mil moléculas de ozono. Al aumentar en la atmosfera los compuestos que degradan el ozono, como su velocidad de formación es lenta, su concentración disminuye hasta que se alcance un nuevo equilibrio entre la velocidad de formación y la degradación ${ }^{12}$.

El estudio de este tema les significó recibir el Premio Nobel de química, en 1995, a MJ Molina, FS 
Rowland y PJ Crutzen. Diez años antes, J Farman, B Gardiner y J Shanklin, reportaron en la revista Nature, registros de niveles bajísimos de ozono, disminuciones de más de 50\% durante la primavera antártica, desde 1980 hasta la publicación ${ }^{15}$. Como sabemos, este fenómeno se ha seguido registrando regularmente sobre la Antártica en los meses de septiembre y octubre hasta la fecha. Los medios de comunicación bautizaron el suceso como de agujero de ozono. Es un nombre poco preciso, ya que la capa de ozono no desaparece sino que se adelgaza considerablemente. Se habla de agujero de ozono cuando los valores de la columna de ozono son menores a $220 \mathrm{UD}^{16}$. Este fenómeno ocurre sólo en la Antártica y se produce por las condiciones climáticas excepcionales que se dan a fines del invierno antártico. Las frías y fuertes comientes de aire, menores a $-80^{\circ} \mathrm{C}$ de este a oeste, permiten la formación de nubes polares altas, en cuya superficie se forman cristales de hielo que facilitan la destrucción acelerada de ozono, incluso por gases que en otras condiciones no reaccionarían con esta molécula. La destrucción catalítica del ozono es tan rápida que en pocas semanas se puede destruir más de 70\% del ozono sobre la Antártica ${ }^{14,16}$.

Pero, zqué ha pasado con la capa de ozono en las últimas décadas sobre el resto del mundo? Hoy en día está bien documentada la declinación de la columna de ozono durante las décadas 1980-89 y 1990-99. Basados en los datos satelitales de la columna de ozono registrados por TOMS (Total Ozone Mapping Spectrometer) y mediciones en tierra, se ha podido documentar, desde aproximadamente 1980, una tendencia a la disminución del ozono estratosférico en las latitudes medias y polares de 3-6\% por década. No se trata tampoco de una disminución homogénea, sino que en invierno es de 6-7\% por década y sólo $3 \%-2 \%$ en verano/otoño $14,17-19$.

A través de la prensa y otros medios de difusión, estas comunicaciones tuvieron un impacto muy grande en la comunidad intemacional y, como respuesta, se produjeron al menos dos consecuencias importantes. En primer lugar, los países desamollados reaccionaron prontamente en lo que fue la primera acción conjunta internacional para enfrentar un problema ecológico mundial y se formalizó su cooperación para restringir y, eventualmente, suprimir la producción de SDO, en la Convención de Viena de 1985 y en 1987 con el Protocolo de Montreal. La otra consecuencia de importancia fue que las investigaciones en fotocarcinogénesis recibieron un nuevo impulso.

\section{IMPACTO DE LA CAPA DE OZONO EN CÁNCER DE PIEL}

Veamos entonces cómo se relaciona el adelgazamiento de la capa de ozono y cáncer de piel mediante el análisis de tres preguntas:

- ¿Cuál es el riesgo cuantitativo de aumento de incidencia de cáncer de piel en función de la depleción de ozono?

- ¿Qué impacto tendrá la disminución de la capa de ozono en cáncer de piel?

- ¿Qué impacto ha tenido la disminución de la capa de ozono en el aumento de la incidencia de cáncer de piel observada en los últimos veinte años?

¿Cuál es el riesgo cuantitativo de aumento de incidencia de cáncer de piel en función de la depleción de ozono? La radiación ultravioleta es el factor de riesgo ambiental más importante para el desamollo de cáncer de piel y, debido a que el ozono absorbe radiación ultravioleta $B$ en su paso por la atmosfera, la disminución de ozono va a aumentar la radiación ultravioleta que llega a nivel de la tierra. Este incremento de radiación ultravioleta debido a la depleción de ozono se denomina "factor de amplificación de radiación" 20 . A través de la red de estaciones Robertson-Berger, de monitoreo de radiación ultravioleta en tierra y mediciones satelitales de ozono (TOMS), se ha llegado a establecer que $1 \%$ de disminución de la columna de ozono, aumenta 1-2\% la radiación ultravioleta $\mathrm{B}$ que llega a la superficie de la tierra ${ }^{21-24}$.

Por otra parte, el aumento de la incidencia de cáncer de piel en función del aumento de la radiación ultravioleta $\mathrm{B}$ carcinogénica, se denomina "factor de amplificación biológico"20,25. Para determinar el factor de amplificación biológico, se requiere conocer la dosis carcinogénica de radiación ultravioleta. Evidentemente, la dosis carcinogénica no puede determinarse experimentalmente en seres humanos y por esto los valores que usamos se determinan extrapolando las dosis carcinogénicas observadas en modelos experimentales (ratas sin pelo), con los parámetros de ajuste en relación a las diferencias de grosor de la epidermis murina y humana ${ }^{26-28}$.

Al relacionar ambos factores, el de amplificación de la radiación y el de amplificación biológico, se obtiene que por cada $1 \%$ de disminución del ozono, el riesgo cuantitativo de desarrollar cáncer de piel aumenta: 3\%-4,6\% para el carcinoma espinocelular y 
1,7\%-2,7\% para el carcinoma basocelular ${ }^{14,25,29,30}$. Para el melanoma maligno, las estimaciones de amplificación son, dependiendo del espectro de acción que se considere, de $1,2 \%$ si se parece al del carcinoma espinocelular o de $0,1 \%$ si se considera como proceso que depende principalmente de radiación ultravioleta $A^{25,31}$. El melanoma maligno es complejo de analizar, ya que no existe un buen modelo experimental. Sólo se ha podido desarmollar experimentalmente en un pez tropical híbrido (xiphophorus) en el que el espectro de acción para melanoma muestra una fuerte dependencia en rango UVA, muy distinto al espectro de acción observado en ratas para inducir carcinoma espinocelular $^{27,32}$. Por este motivo, cualquier modelo cuantitativo de inducción de melanoma maligno por radiación ultravioleta carece de certezas y las proyecciones para melanoma maligno en función de la depleción de ozono deben ser consideradas con reservas $^{33}$. La etiología del melanoma maligno probablemente involucra múltiples procesos con cambios moleculares inducidos, tanto por radiación ultravioleta $\mathrm{B}$, como por radiación ultravioleta $\mathrm{A}$ en la etapa de iniciación y promoción tumoral.

¿Qué impacto tendrá la disminución de la capa de ozono en cáncer de piel? Para responder a esta pregunta, se realizan estudios de escenarios que deben considerar toda la cadena de eventos involucrados como: la producción y emisión de SDO, la depleción de la capa de ozono, la radiación ultravioleta B ambiental, la exposición de la población y relacionarlo con las dosis carcinogénicas observadas en modelos experimentales. Los últimos estudios de escenario, además, consideran los aspectos dinámicos de la acción carcinogénica y el tiempo de latencia en la aparición clínica de cáncer de piel. De lo anterior, se desprende fácilmente que todos estos estudios corresponden a escenarios idealizados y teóricos que no pueden evaluar variables factores relevantes, como los cambios en la conducta humana y naturales impredecibles, como el efecto invernadero, los que pueden agravar o disminuir los futuros efectos sobre la capa de ozono y cáncer de piel.

Sin embargo, llevar a cabo este tipo de estudios ha sido importante y necesario pues han servido para poder cuantificar y comparar la eficacia de las políticas intemacionales sobre restricción de SDO y para enfatizar el trabajo de las campañas de preven- ción de cáncer de piel. Es así como en los primeros estudios a $44^{\circ}$ latitud norte en USA y en Europa, en el escenario sin restricción de SDO, para el año 2100, la incidencia de cáncer de piel no melanoma se cuadruplicaba y, cumpliendo los acuerdos del Protocolo de Montreal, la incidencia se duplicaba ${ }^{31,34}$. De estos resultados alarmantes surgió la imperiosa necesidad de hacer modificaciones más rigurosas con respecto a la producción y emisión de $\mathrm{SDO}$, las que se fuemon concretando en las sucesivas enmiendas al Protocolo de Montreal de 1990 Londres, 1992 Copenhagen, 1997 Montreal y 1999 Beijing.

En los últimos estudios de escenario, en los que además se ha considerado el peníodo de latencia en la aparición de cáncer de piel y el aspecto dinámico de la fotocarcinogénesis, se calcula un exceso de casos adicionales por la depleción de ozono para la década 2050-59 de alrededor de 90 casos por millón de habitantes por año ${ }^{34}$. Este riesgo probablemente es conservador por los siguientes motivos: presume el total cumplimiento en todo el mundo de las restricciones a la emisión de SDO; el envejecimiento de la población probablemente aumentará el exceso de riesgo; no se considera que la población pueda aumentar aún más la exposición solar y, por último, el riesgo es mayor para trabajadores al aire libre con pieles tipo I y II (pieles muy blancas, sensibles a la radiación ultravioleta y que no se broncean 0 mínimo) y para las poblaciones de áreas con mayor depleción de ozono que el promedio.

¿Qué rol ha tenido la disminución global de ozono en el aumento de la incidencia de cáncer de piel observado en todo el mundo? Esta pregunta sólo se puede contestar mediante análisis matemáticos, que se basan en la disminución de ozono por década determinada por la Organización Mundial Meteorológica y la UNEP (United Nations Environmental Programme). Aplicando el factor de amplificación final anteriormente mencionado se esperańa, a $45^{\circ}$ latitud norte, un aumento por década de $11,6 \%$ para el carcinoma basocelular y $21,6 \%$ para el carcinoma espinocelular. Según datos estadísticos, el aumento ha sido considerablemente mayor, de $+82 \%$ para el carcinoma basocelular y $+76 \%$ para el carcinoma espinocelular ${ }^{14}$. Por lo que, probablemente, el aumento de cáncer de piel observado en los últimos veinte años se deba mayoritariamente a una mayor exposición al sol por razones sociales, más que a la baja de los valores de la columna de ozono. 
Pero, ¿qué sucede en las áreas con mayor depleción de ozono que el promedio? Por ejemplo, ¿qué sucede en el extremo más austral de Sudaménca, que ha estado expuesto durante algunos días de septiembre y octubre a valores muy bajos de columna de ozono, incluso han habido días en que el agujero de ozono se ha extendido hacia la punta del continente, durante los últimos 20 años y, concretamente ha afectado a la población de Punta Arenas, Ushuaia y las Islas Malvinas? Es indiscutible que algunos días de septiembre y octubre de los últimos veinte años, estas áreas específicas han recibido dosis mayores a las usuales para estas latitudes con niveles de radiación UVB propios de latitudes subtropicales $^{35}$. Sin embargo, este riesgo ambiental mayor durante ciertos días del año, se ve aminorado debido a que las bajas temperaturas de la zona no favorecen las actividades recreativas al aire libre o usualmente exigen que las personas estén más protegidas por ropa que en la zona central y norte del país. Por este motivo, el incremento de radiación ambiental potencialmente enitematógena y carcinogénica en el extremo sur de nuestro país, durante ciertos días del año, no conlleva el mismo riesgo implícito que esos mismos niveles de radiación ultravioleta $B$ en la zona central y norte del país, donde la población continúa exponiéndose exageradamente a la radiación solar. Por otra parte, la exposición a dosis altas e intermitentes de radiación ultravioleta B que producen quemaduras solares, puede ser muy importante, ya que este patrón de exposición al sol es el que, desde el punto de vista epidemiológico, se asocia con el desarmollo

\section{REFERENCIAS}

1. MiUER D, WeINSTOCK M. Nonmelanoma skin cancer in the United States: incidence. J Am Acad Dermatol 1994; 30: 774-8.

2. De GruJjL FR. Skin cancer and solar UV radiation. Eur J Cancer 1999; 35: 2003-9.

3. Zemeiman V, Roa J, Díaz C, Araya I, Zamaloa G, Faúndez E. Aumento de la incidencia de cáncer cutáneo en hospitales públicos de la región metropolitana (19921998). Rev Chilena Dermatol 2001; 17: 180-5.

4. Zambrano M, Zemeiman V. Estudio epidemiológico del cáncer cutáneo en el Hospital Sótero del Río (19922000). Rev Chil Dermatol 2004; 20: 13-8.

5. ABARCA JF, CASICCIA CC. Skin cancer an ultraviolet B radiation under the Antartic ozone hole: Southem de melanoma maligno cutáneo, especialmente cuando se trata de personas a las que el clima de la zona tampoco les permite desarmollar los mecanismos cutáneos de adaptación al sol ${ }^{36,37}$.

\section{MENSAJES FINALES}

Los efectos carcinogénicos de la radiación ultravioleta tienen una latencia de dos a tres décadas, por lo que el incremento de cáncer de piel en las últimas dos décadas se puede atribuir sólo en parte a la disminución de la capa de ozono y los estudios estadísticos indicarían que el factor más importante sigue siendo la exposición exagerada al $\mathrm{sol}^{7-9}$.

Los niveles ambientales de radiación ultravioleta no se traducen directamente a dosis personales de radiación ultravioleta y a efecto biológico, el comportamiento humano y la capacidad de adaptación siguen siendo los factores más importantes. Por lo que es razonable pensar que con modificaciones menores de la conducta humana, que no son costosas ni mandan mucho esfuerzo, el riesgo esperado de incremento de radiación ultravioleta, como consecuencia de la disminución del ozono estratosférico, puede ser mitigado considerablemente para el hombre.

Es importante y necesario continuar potenciando las campañas de educación y prevención de cáncer de piel, tarea en la que todos los médicos y paramédicos deberíamos estar involucrados.

Chile, 1987-2000. Photodermatol Photoimmunol Photomed 2002; 18: 294-302.

6. GIIES GCG, MaRKS R, Foley P. Incidence of non melanoma skin cancer treated in Australia. Br Med J 1988; 296: 13-7.

7. La Vecchia C, Te VC, Mezzanotte G. Descriptive epidemiology of skin cancer in the Swiss Canton of Vaud. Int J Cancer 1988; 42: 811-6.

8. Mc Gregor JM, Hawk JLM. Efectos agudos de la radiación ultravioleta sobre la piel. En: Dermatología en Medicina General. Fitzpatrick. Buenos Aires: Editorial Médica Panamericana, 2001; 163945.

9. DIFFEY BL Analysis of the risk of skin cancer from sunlight and solaria in subjects living in northem Europe, Photodermatol 1987; 4: 118-26. 
10. DIFFEY BL Ozone depletion and skin cancer. BMJ 1992; 304: 1176-7.

11. DIFFEY BL, SAUNDERS PJ. Behauvior outdoors and its effects on personal ultraviolet exposure rate measured using ambulatory data logging dosimeter. Photochem Photobiol 1995; 61: 615-8.

12. NewMan PA. Introduction to stratospheric ozone. In: Stratopheric Ozone. An Electronic Textbook. Todara $\mathrm{R}$, ed. Disponible en: hptt://hypenion.gsfc.nasa.gov. consultado en mayo 2005.

13. De Gruill FR, Van Der Leun JC. Environmental and health: 3. Ozone depletion and ultraviolet radiation. CMAJ 2000; 163: 851-5.

14. URBACH F. Ultraviolet radiation and skin cancer of humans. Photochem Photobiol B 1997; 40: 3-7.

15. Farman JC, GaRdiner BG, Shankin JD. Large losses of total ozone in Antanctica reveal seasonal ClO x / NO x interaction. Nature 1985; 315: 207-10.

16. NEWMAN PA. The antartic ozone hole. In: Stratopheric Ozone. An Electronic Textbook. Todara R, ed. Disponible en: hptt://hyperion.gsfc.nasa.gov. consultado en mayo 2005.

17. Niu X, Frederick JE, Stein ML, Tiao GC. Trends in column ozone based in TOMS data: Dependence on month, latitude and longitude. J Geogphys Res 1992; 97: 14661-9.

18. Madronich S, Mckenzie RL, Bjorn LO, Caidweil MM. Changes in biologically active ultraviolet radiation reaching the Earth's surface. J Photochem Photobiol B 1998; 46: 5-19.

19. World Metereological Organization. Scientific assessment of Ozone depletion: 1994. In: WMO Global research and Monitoring Project. Albritton $\mathrm{DL}, \mathrm{Au}-$ camp PJ, Megie G, Watson RT, eds. Report № 37, WMO 1994, Genova.

20. Bummthaier M, Saizgeber M, Ambach W. Ozone and ultraviolet-B iradiances: experimental determination of the radiation amplification factor. Photochem and Photobiol 1995; 61: 159-62.

21. KERR JB. Evidence of large upwards trends of ultraviolet B radiation linked to ozone depletion. Science 1994; 262: 1032-43.

22. BASHER RE. Ozone-related trends in solar UVB series. J Geophys Res 1994; 21: 2713-6.

23. KeifKens G, De Gruju FR, Van Der Leun JC. Ozone depletion and increase in annual carcinogenic ultraviolet dose. Photochem Photobiol 1990; 52: 819-23.

24. MAdRonICH S, DE GRUIJL FR. Skin cancer and ultraviolet radiation. Nature 1993; 366: 23.
25. De Gruill FR, Longstreth J, Notval M, CuLen AP, Siaper H, KRIPKE ML ET AL. Health effects from stratospheric ozone depletion and interactions with climate change. Photochem Photobiol Sci 2003; 77: 383-9.

26. De GruIJ FR, Forbes PD. UV induced skin cancer in hairless mouse model. Bioessays 1995; 17: 651-60.

27. De GruIIL FR, VAn Der Leun JC. Estimate of the wavelength dependency of ultraviolet carcinogenesis in humans and its relevance to the risk assessment of a stratospheric ozone depletion. Health Phys 1994; 67: 319-25.

28. De Grujl FR, Sterenborg HJ, Forbes PD, Davies RE, Cole C, KelFKens G et al. Wavelength dependence of skin cancer induction by ultraviolet iradiation of albino hairless mice. Cancer Res 1993; 53: 53-60.

29. ARMSTRONG BK. Stratospheric ozone and health. Int J Epidemiol 1994; 23: 873-85.

30. De GruJl FR, Van Der Leun JC. Estimate of the wavelength dependence of ultraviolet carcinogenesis in humans and its relevance to risk assessement of a stratospheric ozone depletion. Health Phys 1994; 67: 319-25.

31. Longstreth J, De GruJlL FR, KRIPKe ML, Abseck S, ArNold F, SLaper HI et aL. Health risks. J Photochem Photobiol B 1998; 46: 20-39.

32. SetLow RB, Grist E, Thompson K, Woodhead AD. Wavelengths effective in induction of malignant melanoma. Proc Natl Acad Sci USA 1993; 90: 6666-70.

33. LoNGSTRETH JD. Melanoma genesis: putative causes and possible mechanisms. In: Balch $\mathrm{CM}$, Houghton AN, Sober AJ, Soong SJ. Cutaneous Melanoma. St. Louis MO: Quality Medical Publishing, 1998; 535-50.

34. Slater H, Veiders GJ, Danieis JS, De Gruill Fr, Van Der LruN JC. Estimates of ozone depletion and skin cancer incidence to examine the Vienna Convention achievements. Nature 1996; 384: 256-8.

35. Abarca J, Casiccia C, Zamorano F. Increase in sunbums and photosensitivity disonders at the edge of the Antartic ozone hole, Southem Chile, 1986-2000. J Am Acad Dermatol 2002; 46: 193-9.

36. Eiwood J, GaLAGHer R, HiL G. Cutaneous melanoma in relation to intermitent and constant sun exposurethe Westem Canada Melanoma Study. Int J Cancer 1985; 35: 427-33.

37. Holman C, Armstrong B, Heenan P. Relationship of cutaneous malignant melanoma to individual sunlight-exposure habits. J Natl Cancer Inst 1986; 76: 403-14. 